\title{
Performance Evaluation of 802.11 Broadcasts for A Single Cell Network With Unsaturated Nodes
}

\author{
Ashwin Rao ${ }^{1}$, Arzad Kherani ${ }^{2}$, and Anirban Mahanti ${ }^{1}$ \\ 1 Amar Nath and Shashi Khosla School of Information Technology, \\ Indian Institute of Technology Delhi, New Delhi, India \\ ashwin@it.iitd.ac.in, mahanti@cse.iitd.ernet.in \\ ${ }^{2}$ General Motors - India Science Lab, \\ International Technology Park, Bangalore, India \\ arzad.kherani@gm.com
}

\begin{abstract}
Broadcast communication plays a critical role in ad hoc networks especially vehicular communication where a large number of applications are being envisioned to use the broadcast services of IEEE 802.11p. These safety applications exchange messages at very low rates compared to voice and streaming applications but have very stringent latency requirements. The broadcast packets undergoing collision are not retransmitted as the source cannot detect collisions, hence, obtaining the collision probability is critical for the success of applications relying on broadcast communication. In this paper, we initially assume our nodes to be bufferless and model them using a two-state Markov Chain to obtain the collision probability. We then extend our model for finite buffers to study the system under higher data traffic loads.
\end{abstract}

Keywords: Model, 802.11, MAC.

\section{Introduction}

In this paper, we consider an IEEE 802.11 wireless ad hoc network where stations communicate with each other using broadcast messages. The broadcast environment allows receivers to collate information from all transmitting nodes within its neighborhood, allowing receivers to be aware of their immediate surroundings. Currently, "neighborhood awareness" is being leveraged for the design of next-generation vehicle collision warning systems [13/12]. It is envisioned that each vehicle will be equipped with sensors that records its position and dynamics and broadcast this information to nearby vehicles. Each vehicle, in turn, can process the information it receives along with information regarding its own state to detect potential collisions.

The distributed coordinated function (DCF), the primary medium access control (MAC) technique of the IEEE 802.11, is a Carrier Sense Multiple Access with Collision Avoidance (CSMA/CA) scheme designed to support asynchronous data transport where all users have an equal chance of accessing the network. CSMA/CA does not rely on stations detecting a collision by hearing their own transmissions, hence, error-free reception of a packet results in transmission of 
a positive acknowledgment from the receiver. The absence of an acknowledgment is considered to be a failure of successful reception of the packet, resulting in retransmission of the packet; this process of retransmission continues until a threshold number of retransmission attempts are made or until a positive acknowledgement is received. For broadcast transmissions, however, the 802.11 $\mathrm{MAC}$ protocol does not define any retransmission mechanism since receivers do not acknowledge receipt of packets.

The ability of vehicle collision warning systems to prevent imminent collisions depends on the success of broadcast transmissions. Clearly, in a broadcast environment where there exists no direct mechanism to infer the loss of information owing to collisions in the transmission medium, it is important to indirectly and accurately determine the probability of packet collisions. In this paper, we propose an analytic model for IEEE 802.11 broadcast-based ad hoc networks. Specifically, a simple finite-state Markov chain model is developed to model the buffer occupancy process for the IEEE 802.11 MAC considering broadcast traffic within a single cell. The steady state transition probabilities are computed as a function of the packet arrival rate at the MAC layer, the probability of transmission, the number of nodes in the cell and the packet length to obtain the probability of collisions and study the stability of the system.

The remainder of the paper is organized as follows. Section 2 gives an overview of the IEEE 802.11 DCF. The proposed system model is presented in Section 3 . Section 4 provides the simulation setup used and the numerical results obtained from simulations. The related work is discussed in Section 5 in light of our contributions. Conclusions and future work directions are presented in Section 6.

\section{The IEEE 802.11 Medium Access Control}

The Distributed Coordination Function of the IEEE 802.11 MAC provides a time bounded, asynchronous and contention free access protocol based on CSMA/CA 2. A wireless station with a packet to transmit initially monitors the channel activity for the Distributed Inter-Frame Space (DIFS) time period. If the channel is (or becomes) busy during this observation period, the station begins the backoff process. The station then chooses a backoff counter in the range $(0, w)$, where $w$ is the contention window. This backoff time represents a counter that is decremented each time the channel is sensed idle, frozen when transmission is detected in the channel, and decremented when the channel is sensed idle again for more than one DIFS. The station transmits when this counter reaches zero.

The IEEE 802.11 CSMA/CA does not perform collision detection by listening on the channel while its transmission is in progress. Thus, unicast transmissions result in transmission of a positive acknowledgment from the receiver, the absence of which is considered to be a failure of successful reception of the packet at the receiver. The DCF adopts an exponential backoff mechanism in the event of losses. The initial value of $w$ is set to $C W_{\min }$, the minimum contention window, and is doubled after each unsuccessful transmission up to a maximum value $C W_{\max }=2^{m} C W_{\min }$, where $m$ is the maximum backoff stage. 
There are several key differences in how broadcast transmissions are handled with respect to their unicast counterparts. First, RTS/CTS exchange cannot be used in the broadcast environment to reserve channel for transmission. Second, there are no acknowledgements for packet transmissions. Third, there is no retransmission mechanism in place for packets that experience collision especially in vehicular networks due to the stringent latency requirements. Finally, there exists no direct mechanism to detect collisions, and therefore, the protocol does not adapt its sending rate in accordance to the perceived load on the channel.

\section{System Model: Assumptions and Notations}

We consider a single cell of $n$ homogeneous nodes that communicate with each other using the broadcast services of IEEE 802.11. The data payloads exchanged are of the same size and the same data rate is used to broadcast the packets resulting in $L^{\prime}$ busy slots $\left(L^{\prime} \geq 1\right)$. If the DIFS occupies $d^{\prime}$ slots then, on sensing a transmission, the other nodes that have a packet to transmit restart their backoff process after $L=L^{\prime}+d^{\prime}$ slots. The wireless channel is assumed to be noiseless i.e., the errors in packet reception due to fading and other external interferences are not considered as a serious problem compared to the errors caused due to packet collisions. The packet arrivals from the higher layer are assumed to be based on a Poisson distribution. The system being slotted, these packets arrive with a probability $\lambda$ in each slot. In the analysis we initially assume $\lambda$ to be very small $(\lambda<<1)$, such that all the nodes have at most one packet ready for transmission in any given slot resulting in bufferless MAC queues. We later extend this model to higher packet generation rates to model finite buffers at the MAC layer. All the nodes in the network use the same backoff parameters and independently attempt to transmit with a probability $\beta$ in each slot. The summary of the assumptions made are as follows.

1. There are $n$ homogeneous nodes placed in a single cell wireless topology, i.e., the $n$ nodes are able to hear each other.

2. The data exchanged is of a fixed size resulting in busy periods of a fixed number of slots $(L)$.

3. The nodes that have a packet are able to transmit in each slot with a probability $\beta$, i.e., the backoff counter for packet transmission is sampled from a geometric distribution.

4. The arrival rate $\lambda$ is based on a Poisson distribution.

\subsection{Modeling Bufferless MAC}

The wireless channel is seen by all the nodes to be either busy or idle as shown in Fig. 1(a) When the arrival rates low $(\lambda<<1)$, at the end of a busy period, the nodes can either have a backlogged packet ready for transmission or be awaiting an arrival of a packet from the higher layer. Each node at the end of the busy periods can be independently modeled using a two-state Markov Chain as shown 


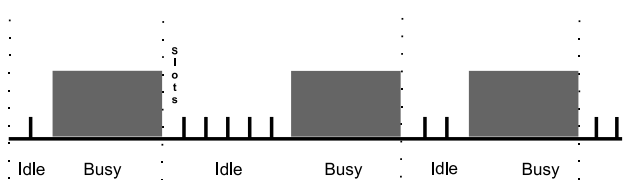

(a) Broadcast of fixed size packets

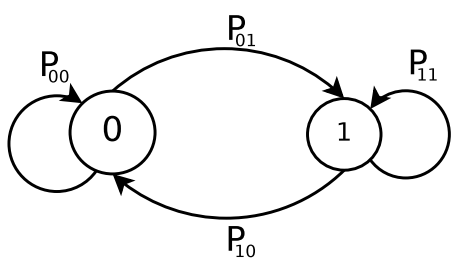

(b) Two state Markov chain for a node

Fig. 1. The state of the wireless channel and the state of the nodes

in Fig. 1(b) State 0 represents the state where the node does not have any packets at the MAC queue while State 1 represents the state where the node has exactly one packet and is performing the backoff process.

At the end of each busy period, the state of each node (either 0 or 1 ) is independent of the state of other nodes present in the system. Let $P_{10}$ denote the steady state transition probabilities from state 1 to state 0 and $P_{10}(k)$ denote the probability of a transition from State 1 to State 0 by a node in a cycle, when there are $k$ other nodes $(0 \leq k \leq n-1)$ contending for the channel.

The value of $P_{10}(k)$ is derived as follows. Consider a marked node that has a packet to transmit at the end of a busy cycle. In the first slot just after a busy period if $k$ nodes other than the marked node have a packet to transmit then, based on the bufferless assumption, there can be $j$ nodes that can receive a packet from the higher layer $(0 \leq j \leq n-k-1)$. The transmit probability $\beta$ is assumed to independent of the state of each of the other $n-1$ nodes in the system, hence, if this node does not transmit, then the next slot can be visualized as the first slot after a busy period in which $k+j$ nodes have a packet to transmit. $P_{10}(k)$ can be given as

$$
P_{10}(k)=\sum_{j=0}^{n-k-1}\left(\begin{array}{c}
n-k-1 \\
j
\end{array}\right) \lambda^{j}(1-\lambda)^{n-k-1-j}(1-\beta)^{k+1} P_{10}(k+j)+\beta .
$$

Similarly, let $P_{01}$ denote the steady state transition probability that a node at State 0 at the beginning of the cycle ends up in State 1 at the beginning of the next cycle and $P_{01}(k)$ denote the probability of that a node undergoes a transition from State 0 to State 1 when there are $\mathrm{k}$ nodes $(0 \leq k \leq n-1)$ contending for the channel. $P_{01}(k)$ can be given as follows.

$$
\begin{aligned}
P_{01}(k)= & \left(1-(1-\beta)^{k}\right)\left(1-(1-\lambda)^{L}\right)+ \\
& \left\{\sum_{j=0}^{n-k-1}\left(\begin{array}{c}
n-k-1 \\
j
\end{array}\right) \lambda^{j}(1-\lambda)^{(n-k-1-j)}(1-\beta)^{k}\right. \\
& \left.\left((1-\lambda) P_{01}(k+j)+\lambda P_{11}(k+j)\right)\right\}
\end{aligned}
$$


Let $\pi$ denote the steady state probability that a node has a packet at the beginning of the cycle. As the nodes are assumed to be homogeneous, the steady state value for $\pi$ at each node will be the same. The steady state values of $P_{01}$ and $P_{10}$ can be computed from $P_{01}(k)$ and $P_{10}(k)$ for a given value of $\pi$ as follows.

$$
\begin{aligned}
& P_{01}=\sum_{k=0}^{n-1}\left(\begin{array}{c}
n-1 \\
k
\end{array}\right) \pi^{k}(1-\pi)^{n-1-k} P_{01}(k) \\
& P_{10}=\sum_{k=0}^{n-1}\left(\begin{array}{c}
n-1 \\
k
\end{array}\right) \pi^{k}(1-\pi)^{n-1-k} P_{10}(k)
\end{aligned}
$$

The value of $P_{10}(n-1)$, numerically computed from (11), can be used to compute $P_{10}(k)$ for $n-1 \geq k \geq 0$ that can be substituted in (44) to give $P_{01}$. As $\pi$ is the steady state probability that a node has a packet to transmit at the end of the busy period, the value of $\pi$ can be obtained as follows.

$$
\pi=\frac{P_{01}}{P_{01}+P_{10}}
$$

Using this value of $\pi$, that can be numerically computed using $P_{01}$ and $P_{10}$, we shall now obtain the probability of packet collisions. At the end of the busy cycle, a node independently attempts a transmission in each slot with a probability $\pi \beta$. The packet transmitted is successfully received by all the other nodes only if none of the other nodes attempt a transmission in the same slot. Hence the probability of successful transmission is given by $(1-\pi \beta)^{n-1}$ and the probability of collisions as the fraction of attempted transmissions undergoing collisions is

$$
P_{\text {coll }}=1-(1-\pi \beta)^{n-1} .
$$

The system parameters $\lambda, \beta, L$ and $n$ are used to obtain the values of $\pi$ and the probability of collision $P_{\text {coll }}$. The real world implications of these parameters are as follows.

1. The packet arrival rate $(\lambda)$ abstracts the packet generation process at the application layer.

2. The probability of transmission in a given slot $(\beta)$ represents the contention window of IEEE 802.11.

3. The length of the busy period $(L)$ can be used to abstract the packet length and the data transmission rates.

4. The number of nodes present in the single cell $(n)$ represents the transmission range of the nodes.

\subsection{Modeling MAC with Finite Buffers}

We now look at the MAC layer with finite buffers where packets are queued up according to the drop tail queuing principle. Arrivals when a node has a packet to transmit are queued up provided the queue contains less than $K$ elements 
$(K \geq 1)$. At the end of a busy period, each node can be modeled as a Markov Chain of $K+1$ states as shown in Figure 2. State $i$ represents the state where the node has $i(0 \leq i \leq K)$ packets in the MAC queue. At the end of the busy period a given node is in State $i$ with a probability $\pi_{i}$. The probability of collision for this system can be given as

$$
P_{\text {coll }}=1-\left(1-\left(1-\pi_{0}\right) \beta\right)^{n-1} .
$$

The transition probabilities from State $i$ to State $j\left(P_{i, j}\right)$, where $i, j \in[0, K]$, can be obtained from $P_{i, j}(k)$ indicating the probability of transition from state $i$ to state $j$ when $k(0 \leq k \leq n-1)$ other nodes have a packet to transmit. From any state $i(0 \leq i \leq K)$, a transition to state $j(\max (0, i-1) \leq j \leq K)$ is possible at the end of a busy period with a probability $P_{i, j}(k)$. One such equation of $P_{i, j}(k)$ for $0<i<K$ and $j \geq i$ is as follows.

$$
\begin{aligned}
P_{i, j}(k)= & (1-\beta)\left(1-(1-\beta)^{k}\right)\left(\left(\begin{array}{c}
L+1 \\
j-i
\end{array}\right) \lambda^{j-i}(1-\lambda)^{L+1-j+i}\right) \\
& +\beta\left(\left(\begin{array}{c}
L+1 \\
j-i+1
\end{array}\right) \lambda^{j-i+1}(1-\lambda)^{L-j+i}\right) \\
& +\sum_{m=0}^{n-k-1}\left(\begin{array}{c}
n-k-1 \\
m
\end{array}\right) \lambda^{m}(1-\lambda)^{n-k-1-m}(1-\beta)^{k+1} \\
& \left((1-\lambda) P_{i, j}(k+m)+\lambda P_{i+1, j}(k+m)\right)
\end{aligned}
$$

We know from the analysis of bufferless system that the probability that a node has a packet to be transmitted in the steady state, $\pi$, depends on the system parameters $n, \beta, L, K$ and $\lambda$. For the finite buffer analysis we relax the constraint of low packet generation rates and assume all possible $\lambda$, i.e, $0<\lambda \leq 1$. Recall that $L$ represents the inverse of the transmission bandwidth. One important problem for such systems is to obtain the number of nodes $n$ the system can support for a given configuration of $\beta, \lambda$ and $L$. The definition of "can support" qualifier above can be subjective. For example, one may state a value for probability of MAC layer collision and another value of packet blocking probability (the probability with which new arriving packets are lost) and then find maximum value of $n$ that satisfies both these constraints. We shall be attempting to understand this problem from the point of view of system stability. For this, we assume that each node has a storage capacity of $K$ units. The buffer size $K$ will now serve as another system parameter that can be tuned to support a target number of nodes. To do this, we need an understanding of dependence of various performance metric on the system parameters. Given the complex system, we shall attempt an approximate analysis which gives a good approximation.

Approximate Analysis: Let $\pi_{K}(k), 0 \leq k \leq K$ be the probability that a marked node is having $k$ packets in its buffer in the steady state when the buffer size at the node is $K$ packets. The probability that an arriving packet is blocked is then $\pi_{K}(K)$. Recall the time instants at which a transmission just got over in the network. One can write down transition probability equations for the process of 


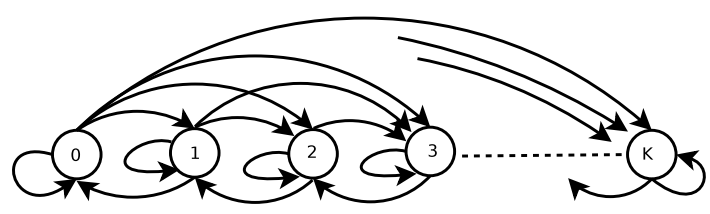

Fig. 2. Markov Chain for a node with finite buffer

buffer occupancy of a marked node (embedded at the ends of successive network transmissions). These equations will be approximate as we make the assumption that the state of all the nodes in the system are independent of each other at the ends of successive transmissions thus, marked node would see $k$ other nodes with at least one packet in their buffers with probability $\left(\begin{array}{c}n-1 \\ k\end{array}\right)\left(1-\pi_{K}(0)\right)^{k} \pi_{K}(0)^{n-1-k}$

Stability Analysis: Let us now assume that the nodes have infinite buffering capacity, i.e., $K=\infty$. We are interested in ensuring stability of buffer occupancy processes, i.e., that the buffer occupancy process converges over time (in law) to a random variable with finite expected value.

A family of probability measures over positive real line $\left\{P_{j}(\cdot)\right\}_{j}$ is called tight if for any given $\eta>0$, one can find $C(\eta)<\infty$ such that $\int_{y=C(\eta)}^{\infty} d P_{j}(y)<\eta$ uniformly for all values of $j$. We state the following theorem (proof follows standard tightness arguments),

Theorem 1. For a given set of system parameters $\lambda, n, K$ and $\beta$, the buffer occupancy process of any node is stable iff the sequence $\left\{P_{K}(\cdot)\right\}_{K}$ forms a tight sequence of probability measures.

Corollary 1. For a given set of system parameters $\lambda, n, K$, and $\beta$, the buffer occupancy process of any node is stable iff $\pi_{K}(K) \rightarrow_{K \rightarrow \infty} 0$.

Proof: Let the sequence $\left\{P_{K}(\cdot)\right\}_{K}$ be tight. For any given $\eta>0$, there exits a $C(\eta)<\infty$ such that $\sum_{j=C(\eta)}^{K} \pi_{K}(K)<\eta$ for all $K>C(\eta)$. This is possible if and only if $\pi_{K}(K) \rightarrow K \rightarrow \infty 0$.

\section{Numerical Results and Discussions}

A simple discrete event simulator to simulate the state of the nodes and the wireless channel for $10^{6}$ cycles of busy and idle periods was written. The system parameters used were obtained as follows. The slot duration of IEEE 802.11 is 20 $\mu \mathrm{s}$ [2] while data rate vehicular communication is $6 \mathrm{Mbps}$ [137]. The number of slots $L$ required to transmit a a signed message of 250 bytes [1] $=\frac{250 * 8}{6 * 10^{6} * 20 * 10^{-6}} \approx$ 17. The awareness messages are broadcast once every 100 milliseconds [313], i.e., once every $\frac{100 * 10^{-3}}{20 * 10^{-6}}=5000$ slots with a transmission range of about 150 meters [13]. Assuming each vehicle, of length 4 meters, maintains a distance of 2 meters with the vehicle in front of it, we can have 150/6 $=25$ vehicles in each lane giving approximately 150 nodes in a six lane freeway lying in the 
Table 1. Simulation Parameters

\begin{tabular}{|l|l|}
\hline Parameter & Value \\
\hline Number of nodes $(n)$ & 3 to 200 \\
\hline $\begin{array}{l}\text { Probability of transmit } \\
\text { in a given slot }(\beta)\end{array}$ & $1 / 16$ and $1 / 8$ \\
\hline $\begin{array}{l}\text { Probability of packet arrival } \\
\text { in a given slot }(\lambda)\end{array}$ & $1 / 2500$ and $1 / 5000$ \\
\hline Number of busy slots $(\mathrm{L})$ & 25,50 \\
\hline
\end{tabular}

transmission range of a given node. Using the above information, the simulation parameters given in Table 1 were used for studying the behavior of the system. Figure 3 and Figure 4 show the relation of $\pi$ and the system parameters $\lambda, \beta, n$ and $L$ for the bufferless nodes. Figure $3(\mathrm{a})$ shows the relation between $\beta$ and $\pi$ when $L$ and $\lambda$ are kept constant at 25 and $1 / 5000$ respectively. An increase in value of $\beta$ from $1 / 16$ to $1 / 8$ shows a decrease in the value of $\pi$. Higher values of $\beta$ indicate lower number of slots for which a node remains in State 1 when the channel is idle, resulting in lower values of $\pi$.

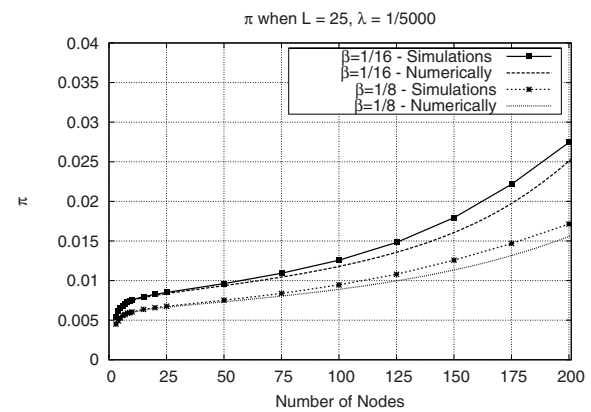

(a) Relation between $\pi$ and $\beta$

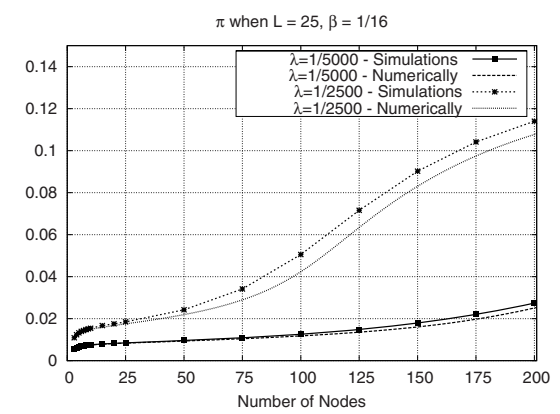

(b) Relation between $\pi$ and $\lambda$

Fig. 3. Impact of $\beta$ and $\lambda$ on $\pi$

Figure 3(b) shows the relation between $\lambda$ and $\pi$ when $L$ and $\beta$ are kept constant at 25 and $1 / 16$ respectively. An increase in value of $\lambda$ from $1 / 5000$ to $1 / 2500$ shows an increase in the value of $\pi$. This can be explained as follows. Higher values of $\lambda$ indicate an increase in the arrival rate resulting in an increase in the number of $P_{01}$ transitions. Figure 3(a) and Figure 3(b) show that $\pi$ is more sensitive to $\lambda$ as compared to $\beta$, hence, adapting the packet arrival rates from the higher layers has greater impact on $\pi$ compared to adapting the transmit probabilities at the MAC layer.

Figure 4(a) shows the relation between $L$ and $\pi$ when $\lambda$ and $\beta$ are kept constant at $1 / 5000$ and $1 / 16$ respectively. An increase in value of $L$ from 25 to 50 shows an increase in the value of $\pi$. This can be explained as follows. Higher values of $L$ indicate an increase in the number of slots for which the channel 
is kept busy, resulting in increase in the number of slots required to complete a cycle, as shown in Figure 1(a). These extra slots account for the increased number arrivals in the cycle resulting in higher values of $\pi$. Figure $3(\mathrm{~b})$ and Figure 4(a) shows that an increase in the value of $L$ is similar to an increase in the value of $\lambda$, hence, for fixed packet sizes increasing the data rate can be used to reduce the values of $\pi$ and thus packet collisions. Figure 4(b) shows the that $P_{\text {coll }}$ increases as the number of nodes contending the wireless channel increase.

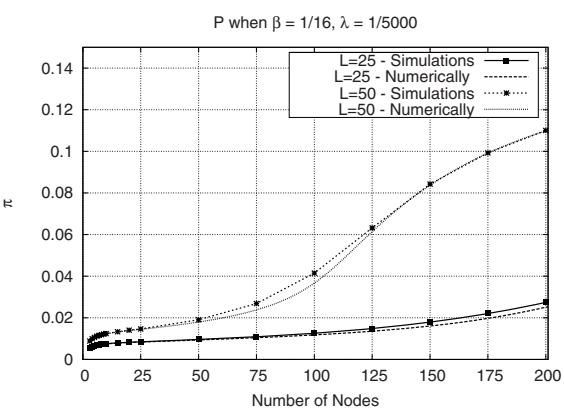

(a) Relation between $\pi$ and $L$

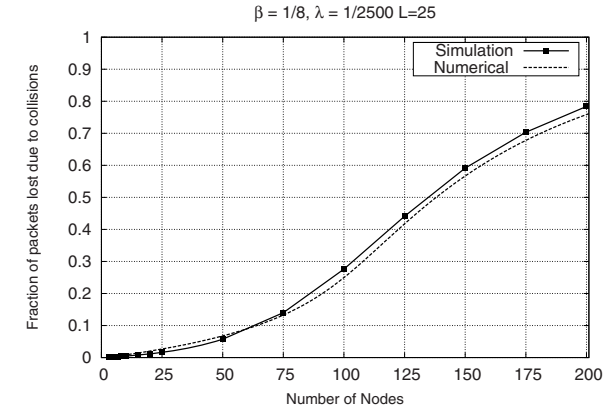

(b) Fraction of packets lost due to collisions

Fig. 4. Impact of $L$ on $\pi$ and $n$ on $P_{\text {coll }}$

Figure 5 illustrates the behavior of the system of finite buffers. Figure $5(\mathrm{a})$ shows the $\pi_{i}$ values for $(0 \leq i \leq K)$ when the buffer size $K$ is 4 for $\lambda=1 / 1000$, $\beta=1 / 16$ and $L=25$. The value of $\pi_{k}$ converges to a fixed value as the number of nodes increase. The value of $\pi_{0}$ converges to a value greater than 0.5 indicating that the buffers are empty for a considerable amount of time even when the packet arrival rate is as high as $1 / 1000$. The stability of the system is reflected in Figure 5(b) which shows the relation between $\pi_{K}$ and $K$. Even for high arrival rates, i.e., when $\lambda=1 / 100$, i.e, packets generated once every 2 milliseconds, we see that the system can support about 200 nodes for buffer sizes less than 10 . The

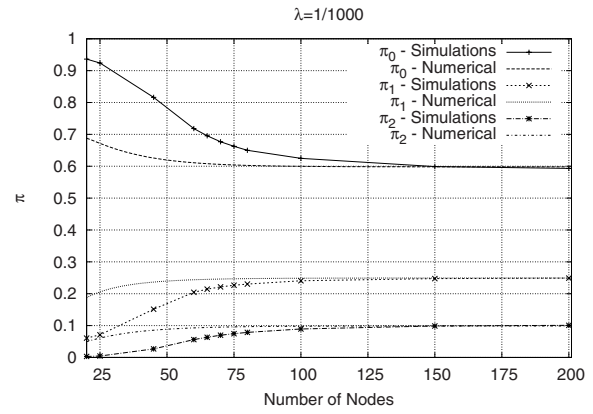

(a) $\pi_{k}$ when $K=4$

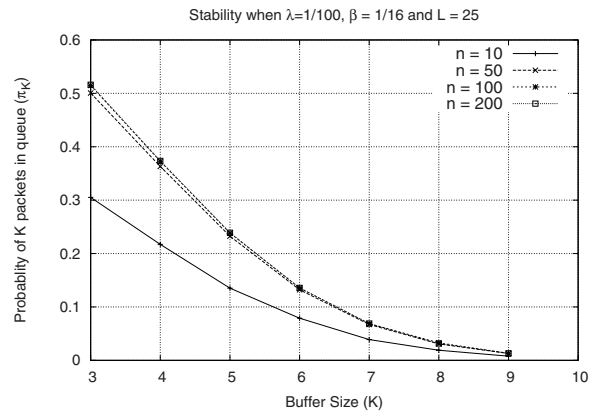

(b) Stability of the system with respect to $n$

Fig. 5. System with finite buffers 
absence of retransmissions results in the goodput to be $1-P_{\text {coll }}$ for the single cell, hence, even if the system is stable, Figure 4(b) shows that the goodput reduces considerably as the number of nodes increase.

This behavior is interesting because the absence of retransmissions imply that large collision probability is good for the stability of the system which is not the case when there are retransmissions. Large collision probabilities are a result of significant number of backlogged nodes resulting in an increase in the waiting time for transmission of the packets. The interplay of these two effects make the stability analysis of these systems complex and we are currently working on it. Numerical approximations based on our proposals in this paper help in the stability analysis.

\section{Related Work}

Modeling wireless networks especially those based on the IEEE 802.11 standard has received considerable research attention over the years. Given the space constraints a detailed discussion is not feasible, hence, we limit ourselves to the work that allows us place our contribution in the overall context.

In this paper we model the buffer occupancy process for broadcast networks where there are no retransmissions. Retransmissions exist in unicast communication, hence, modeling of the backoff process essential for analysis of unicast communication. In the seminal paper for performance analysis of 802.11 DCF 4] and its extensions [8], the backoff process is modeled as a Markov Chain for nodes with saturated MAC queues to obtain the collision probability. The model by Bianchi et al. [4 cannot be used to study the stability of the system as their model assumes saturated MAC queues.

Malone et al. [10] extend [4] for unsaturated MAC queues for unicast networks. Similarly Chen et al. 5] provide a model based on the one available in [4] for broadcast traffic assuming saturated MAC queues. The above proposals model the backoff process while this work models the buffer occupancy process to study the impact of packet arrival rates from higher layer, packet transmission probabilities at the MAC layer, packet transmission times and number of neighbors present in the broadcast network on the packet collision probabilities when the MAC can buffer only a finite number of packets.

Qui et al. [11] study the interference in wireless networks relying on broadcast and unicast communication, but, they do not address any stability issues. Choi et al. 6] provide an analytical model to study the hidden node problem in multi-hop networks by providing a two-state Markov Chain for modeling the communication channel. Similarly, we model the channel to be either idle or busy in any given cycle and nodes to have at most one packet in our initial analysis. Torrent-Moreno et al. 1415] provide insights into the impact of radio propagation models using the probability of reception as a performance metric. We believe our work can be extended to accommodate the channel models considered in 14[15]. 


\section{Conclusion}

In section 3 we provide equations for the transition probability and $\pi$. A closed form of the equations is desirable for the sensitivity and stability analysis of this system. Simulations show that adapting the packet arrival rates has a greater impact than adapting the transmit probabilities as $\pi$ is more sensitive to $\lambda$ than $\beta$ under the simulated parameters. Similarly an increase in the value of $L$ results in an increase in $\pi$ hence increasing in data transmission rates or reducing packet sizes can result in lower collisions in broadcast networks. The region where these observations hold true can be obtained only after obtaining the closed form of $\pi$. Similarly a closed form expression for finite buffers needs to be derived. The current equations for $\pi_{k}$ are quadratic hence an algorithm needs to be derived to obtain the desired root of the equation.

In this paper we target the performance evaluation of IEEE 802.11 broadcast networks. A simple two-state Markov chain is as used to model the system under low loads. The model is further extended to support higher traffic loads. The proposed models give a good insight into the working of the system that can be used for sensitivity and stability analysis. The simulations show that a stable system may not provide the desired goodput and adapting the packet arrival rates has a greater impact than adapting the transmit probabilities, motivating the need for the applications to adapt their rates based on the sensed channel load.

\section{References}

1. IEEE Trial-Use Standard for Wireless Access in Vehicular Environments - Security Services for Applications and Management Messages, IEEE Std 1609.2-2006 (2006)

2. IEEE Standard for Information technology-Telecommunications and information exchange between systems-Local and metropolitan area networks-Specific requirements - Part 11: Wireless LAN Medium Access Control (MAC) and Physical Layer (PHY) Specifications. June 12 (2007)

3. Bai, F., Krishnan, H., Sadekar, V., Holland, G., ElBatt, T.: Towards characterizing and classifying communication-based automotive applications from a wireless networking perspective. In: 1st IEEE Workshop on Automotive Networking and Applications (AutoNet 2006) (2006)

4. Bianchi, G.: Performance analysis of the IEEE 802.11 distributed coordination function. Selected Areas in Communications, IEEE Journal on 18(3), 535-547 (2000)

5. Chen, X., Refai, H.H., Ma, X.: Saturation performance of IEEE 802.11 broadcast scheme in ad hoc wireless lans. In: Vehicular Technology Conference, VTC-2007 Fall. 2007 IEEE 66th (September 30-October 3, 2007), pp. 1897-1901 (2007)

6. Choi, J.-M., So, J., Ko, Y.-B.: Numerical analysis of IEEE 802.11 broadcast scheme in multihop wireless ad hoc networks. Information Networking, 1-10 (2005)

7. DSRC Industry Consortium. DSRC Technology and the DSRC Industry Consortium (DIC) Prototype Team, White Paper. Tech. rep (2005)

8. Gupta, N., Kumar, P.R.: A Performance Analysis of the 802.11. Wireless LAN Medium Access Control Communications in Information and Systems 3, 279-304 (2003) 
9. Kumar, A., Altman, E., Miorandi, D., Goyal, M.: New insights from a fixed-point analysis of single cell IEEE 802.11 WLANs. IEEE/ACM Trans. Netw. 15(3), 588601 (2007)

10. Malone, D., Duffy, K., Leith, D.: Modeling the 802.11 distributed coordination function in nonsaturated heterogeneous conditions. IEEE/ACM Trans. Netw. 15(1), 159-172 (2007)

11. Qiu, L., Zhang, Y., Wang, F., Han, M.K., Mahajan, R.: A general model of wireless interference. In: MobiCom 2007: Proceedings of the 13th annual ACM international conference on Mobile computing and networking, pp. 171-182. ACM, New York (2007)

12. Tan, H.-S., Huang, J.: DGPS-based vehicle-to-vehicle cooperative collision warning: Engineering feasibility viewpoints. Intelligent Transportation Systems, IEEE Transactions on 7(4), 415-428 (2006)

13. The CAMP Vehicle Safety Communications Consortium. Vehicle Safety Communications Project Task 3 Final Report: Identify Intelligent Vehicle Safety Applications Enabled by DSRC. Tech. Rep. 809859, National Highway Traffic Safety Administration, U. S. Department of Transportation (USDOT) (March 2005)

14. Torrent-Moreno, M., Corroy, S., Schmidt-Eisenlohr, F., Hartenstein, H.: IEEE 802.11-based one-hop broadcast communications: Understanding transmission success and failure under different radio propagation environments. In: MSWiM 2006: Proceedings of the 9th ACM international symposium on Modeling analysis and simulation of wireless and mobile systems, pp. 68-77. ACM Press, New York (2006)

15. Torrent-Moreno, M., Jiang, D., Hartenstein, H.: Broadcast reception rates and effects of priority access in 802.11-based vehicular ad-hoc networks, pp. 10-18. 